\title{
Observations of the Li, Be, and B isotopes and constraints on cosmic-ray propagation
}

\author{
G. A. de Nolfo ${ }^{\text {a }}$, I. V. Moskalenko ${ }^{\text {b,a }}$, W. R. Binns ${ }^{\text {c }}$, E.R. Christian ${ }^{\text {d }}$, A. C. Cummings ${ }^{\text {e }}$, \\ A. J. Davis ${ }^{\mathrm{e}}$, J. S. George ${ }^{\mathrm{e}, \mathrm{f}}$, P. L. Hink ${ }^{\mathrm{c}}$, M. H. Israel ${ }^{\mathrm{c}}$, R. A. Leske ${ }^{\mathrm{e}}$, M. Lijowski $^{\mathrm{c}}$, \\ R. A. Mewaldt ${ }^{\mathrm{e}}$, E. C. Stone ${ }^{\mathrm{e}}$, A. W. Strong ${ }^{\mathrm{g}}$, T. T. von Rosenvinge ${ }^{\mathrm{a}}$, \\ M. E. Wiedenbeck ${ }^{\mathrm{h}}$, N. E. Yanasak ${ }^{\mathrm{e}}$ \\ ${ }^{a}$ NASA/Goddard Space Flight Center, Code 661, Greenbelt, MD 20771 USA \\ ${ }^{\mathrm{b}} \mathrm{HEPL} /$ Stanford University, Stanford, CA 94305 USA \\ ${ }^{\mathrm{c}}$ Department of Physics and McDonnell Center for the Space Sciences, Washington University, St. Louis, MO 63130 USA \\ d NASA Headquarters, Washington DC 20546, USA \\ e Space Radiation Laboratory, California Institute of Technology, Pasadena, CA 91125 USA \\ ${ }^{\mathrm{f}}$ The Aerospace Corporation, M2/260, Los Angeles, CA 90009, USA \\ ${ }^{\mathrm{g}}$ Max-Planck-Institut für extraterrestrische Physik, Postfach 1603, D-85740 Garching, Germany \\ $\mathrm{h}^{\mathrm{h}}$ et Propulsion Laboratory, California Institute of Technology, Pasadena, CA 91109 USA
}

\begin{abstract}
The abundance of $\mathrm{Li}, \mathrm{Be}$, and $\mathrm{B}$ isotopes in galactic cosmic rays (GCR) between $\mathrm{E}=50-200 \mathrm{MeV} / \mathrm{nucleon}$ has been observed by the Cosmic Ray Isotope Spectrometer (CRIS) on NASA's ACE mission since 1997 with high statistical accuracy. Precise observations of Li, Be, B can be used to constrain GCR propagation models. We find that a diffusive reacceleration model with parameters that best match CRIS results (e.g. B/C, Li/C, etc) are also consistent with other GCR observations. A 15-20\% overproduction of $\mathrm{Li}$ and $\mathrm{Be}$ in the model predictions is attributed to uncertainties in the production cross-section data. The latter becomes a significant limitation to the study of rare GCR species that are generated predominantly via spallation.
\end{abstract}

Key words: cosmic rays, Galaxy: general, Galaxy: abundances, Galaxy: evolution, ISM: general

\section{Introduction}

The enormous excess of the abundances of $\mathrm{Li}$, $\mathrm{Be}$, and $\mathrm{B}(\mathrm{LiBeB})$ relative to $\mathrm{C}, \mathrm{N}$, and $\mathrm{O}$ in GCRs by a factor of $\sim 10^{4}$ compared with solar system abundances is attributed to the fragmentation of primary GCR nuclei, mainly $\mathrm{C}, \mathrm{N}$, and $\mathrm{O}$, on interstellar $\mathrm{H}$ and He atoms (Freier et al., 1959). Observations of secondary GCRs such as LiBeB thus provide strong constraints on propagation models of GCRs within the Galaxy, since the production of these light isotopes depends on the amount of matter traversed during propagation. B/C ratio is often used to tune propagation parameters, such that the model predictions agree automatically with B/C. Li and Be are particularly interesting since their production depends not only on the interaction of $\mathrm{CNO}$, but also on "tertiary" interac- 
tions in the ISM (e.g. B, Be $\rightarrow$ Li), and therefore may provide further restrictions on propagation models. In particular, one might expect to observe a stronger energy dependence of $\mathrm{Li} / \mathrm{C}$ and $\mathrm{Be} / \mathrm{C}$ ratios compared to the $\mathrm{B} / \mathrm{C}$ ratio. In addition, establishing the details of GCR propagation in the Galaxy will be beneficial for other studies, such as searches for signatures of exotic physics in GCR, spectra and origin of galactic and extragalactic $\gamma$ ray background, nucleosynthesis, and solar modulation (e.g., Moskalenko et al., 2005).

The Cosmic Ray Isotope Spectrometer (CRIS) on the NASA/ACE spacecraft (Stone et al., 1998) measures the isotopic composition of elements with $2 \leq Z \leq 30$ in the energy range from $\sim 30$ $500 \mathrm{MeV} /$ nucleon since 1997 with unprecedented precision. High statistics allows $\mathrm{LiBeB}$ to be studied over an extended energy range $(\sim 50-200$ $\mathrm{MeV} /$ nucleon) for the first time. We present absolute intensities and relative elemental and isotopic abundances of GCR LiBeB observed by CRIS during near solar minimum conditions, and we compare these results with observations from previous instruments. We also discuss the implications of these observations in the context of a GCR transport model, GALPROP. GALPROP has recently been improved to predict $\mathrm{LiBeB}$ GCR isotopic abundances, including re-examined fragmentation cross sections used as input to the model. The precision of the data set from CRIS is high enough (e.g., de Nolfo et al., 2001) to require a reevaluation of the contributing uncertainties in the model calculations, particularly the uncertainties in the isotopic cross-sections.

\section{Intensities and Elemental Abundances}

The absolute intensities of $\mathrm{Li}, \mathrm{Be}, \mathrm{B}$, and $\mathrm{C}$ as measured by CRIS are shown in Fig. 1a (solid circles). The data set considered for this study covers near solar minimum conditions between 1998 Jan. 1 and 1999 Jan. 23. Excluding periods of intense solar activity, the total time period corresponds to 311 days (for details see de Nolfo et al., 2003). The uncertainties shown in Fig. 1a for CRIS observations include both statistical and system- atic uncertainties. The statistical uncertainties are typically small, ranging from $0.5 \%$ for $\mathrm{C}$ to $5 \%$ for $\mathrm{Li}$. The systematic uncertainties result from the various correction factors within the calculation for the absolute intensity such as uncertainties in the determination of the geometry factor $(2 \%)$, spallation loss within the instrument (1-5\%), and the tracking efficiency of the Scintillating Optical Fiber Hodoscope (SOFT) (Stone et al., 1998) $(<1 \%$ for $Z>5$ and up to $6 \%$ for $\mathrm{Li})$.

The elemental and isotopic ratios observed by CRIS are shown in Fig. $1 \mathrm{~b}$ and Fig. 2 for solar minimum conditions. The ratios of the spallogenic nuclei $(\mathrm{B}, \mathrm{Be}$, and $\mathrm{Li}$ ) to mostly primary nuclei such as $\mathrm{C}$ are particularly important in constraining propagation models since these ratios are sensitive to the amount of material traversed by GCRs from the source to detection at Earth. In addition, abundance ratios tend to be less sensitive to instrumental uncertainties than absolute intensities. In Fig. 1b, the experimental error bars include both systematic and statistical uncertainties.

In Fig. 1, CRIS results are compared with previous observations. A direct comparison of CRIS results with previous observations is complicated at these energies by the effects of solar modulation. The effect of solar modulation is approximated by the spherically symmetric model of Fisk (1971), characterized by the solar modulation parameter $\phi$ (Gleeson and Axford, 1968). Levels of modulation are determined for a given source spectrum by matching post-propagation interstellar spectra as predicted by GALPROP to CRIS at low energies and to HEAO-3 at high energies. The average amount of solar modulation experienced by particles observed by CRIS during near-solar minimum conditions corresponds to $\phi \approx 550 \mathrm{MV}$. While the modulation parameter can be used to characterize the amount of modulation experienced during a specific time period, the derived value of $\phi$ also depends on the initial choice of the source spectrum and thus on the propagated interstellar spectrum adopted as input in the solar modulation calculation. For instance, the solar modulation adopted for CRIS during solar minimum is closer to $\phi=$ $\sim 400 \mathrm{MV}$ based on predictions from the Leaky Box Model (Davis et al., 2001; Niebur et al., 2003).

CRIS observations are in good agreement with 

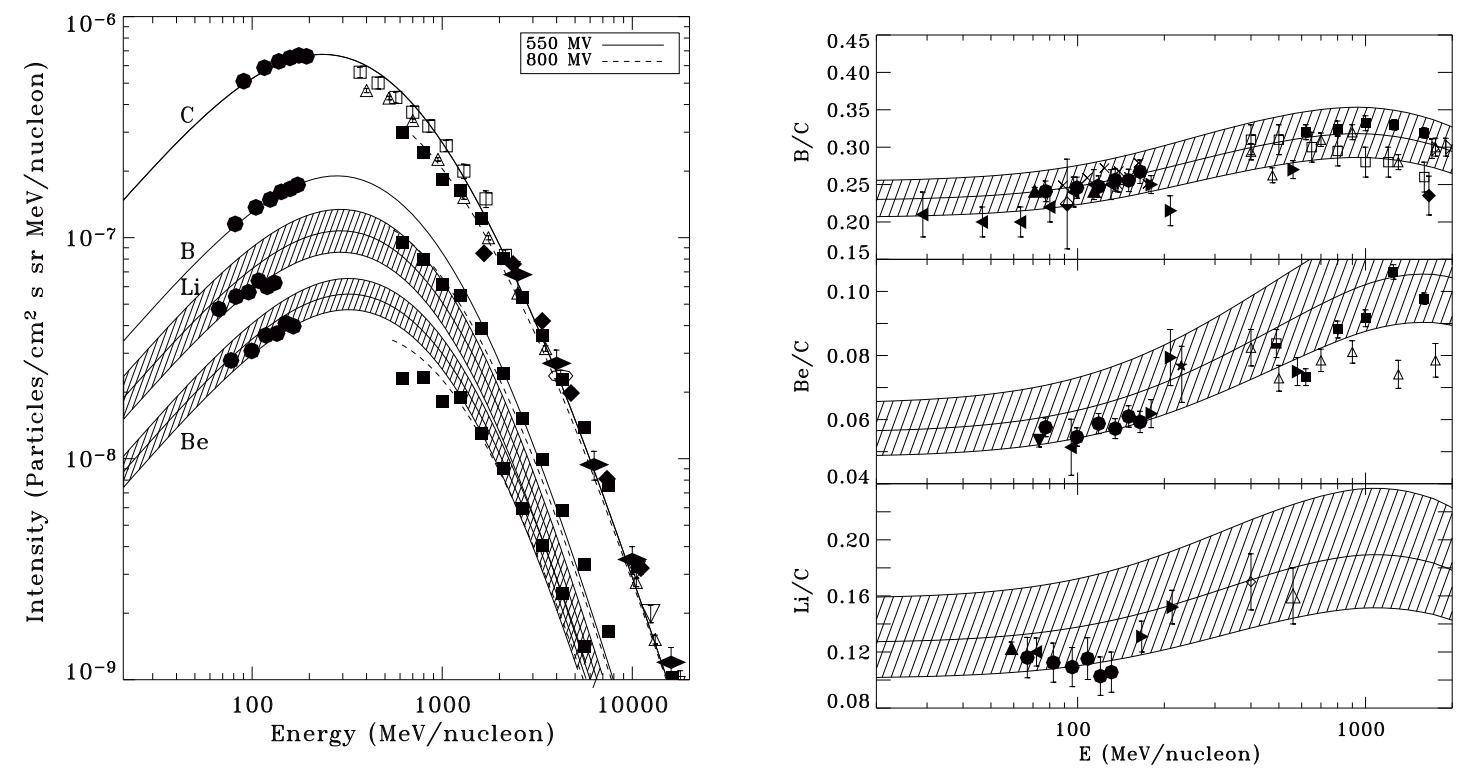

Fig. 1. [a] Intensities of C, B, Be, and Li shown for two levels of solar modulation ( $\phi=550$ (solid curve) and $800 \mathrm{MV}$ (dashed curve)) corresponding to the epoch when the experimental data were taken, and [b] relative elemental abundances compared with previous observations. The experimental data is compared with the results of a propagation model GALPROP. See text for details. (Symbols refer to CRIS: $\bullet$, Maehl et al. 1977: $\square$, Webber et al. 1972: $\triangle$, Englemann et al. 1990: $\mathbf{\square}$, Orth et al. 1978: $\downarrow$, Buffington et al. 1978: $\diamond$, Simon et al. 1980: $\diamond$, Buckley et al. 1994: $\square$, Chapel and Webber 1981: $\nabla$, Lezniak et al. 1978: $\triangle$, Muller et al. 1991: †, Dwyer et al. 1978: $\triangleright$, Webber et al. 1977: $\triangleright$, Garcia-Munoz et al. 1987: ४, Duvernois et al. 1996: $*$, Mewaldt et al. 1981: $\diamond$, Wiedenbeck and Greiner 1980: +, Webber et al. 2002: $\mathbf{\nabla}$, Hagen et al. 1977: $\star$, Fisher et al. 1976: $\boxminus$, Juliusson et al. 1974: +, Garcia-Munoz et al. 1977: O, Connell et al. 2001: ×, Krombel et al. 1988: $\triangleleft$, Lukasiak et al. 1999: $\mathbf{\Delta})$.

the previous observations of Voyager 1, 2 and IMP 7,8 , both of which were made during near solar modulation conditions similar to CRIS. The Voyager data were accumulated over 21 years with a weighted average solar modulation level of $\phi=450$ MV. This may not be a good representation of the actual modulation level experienced over 21 years (Connell et al., 1998) and thus complicates a comparison with CRIS data. At higher energies, the modulation level determined for HEAO-3 data (Englemann et al., 1990) corresponds to $\phi \sim 800$ MV (Davis et al., 2000).

\section{GCR Propagation Calculations}

To interpret the CRIS observations of $\mathrm{LiBeB}$ spectra and isotopic abundances, we use the diffusive reacceleration model (Seo and Ptuskin,
1994). "Reacceleration" is a distributed acceleration of particles due to scattering on random hydromagnetic waves moving at Alfvén speed in the interstellar medium (Fermi 2nd-order mechanism). The model reproduces the peak near 1000 $\mathrm{MeV} /$ nucleon in the ratios of secondary to primary nuclei in a physically motivated way (where the parameters are: normalization and index of the diffusion coefficient, the Alfvén speed, and the size of the galactic halo), and is consistent with the K-capture parent/daughter nuclei ratio (Jones et al., 2001a; Niebur et al., 2003).

While the diffusive reacceleration model successfully reproduces data on GCR nuclear species (Moskalenko et al., 2002), it underproduces GCR antiprotons by a factor of $\sim 2$ at $2 \mathrm{GeV}$, which may be a signature of new effects. In particular, the propagation of low-energy particles may be aligned to the magnetic field lines instead of isotropic 


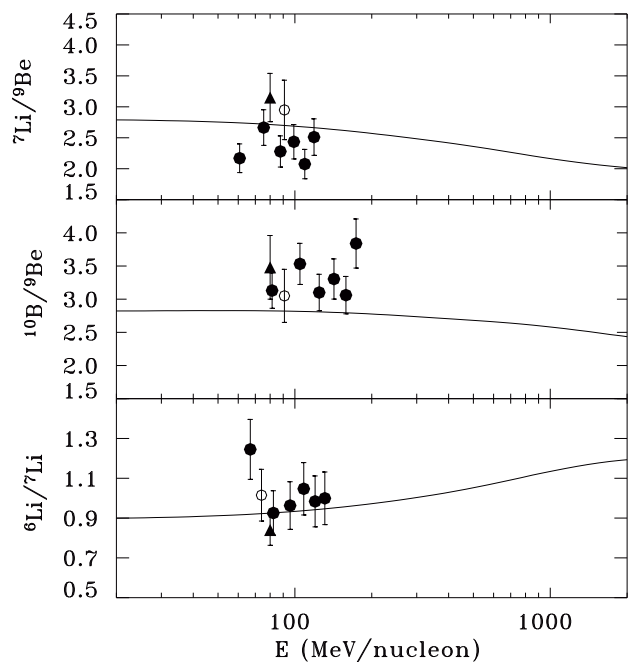

Fig. 2. Isotopic ratios of ${ }^{6} \mathrm{Li} /{ }^{7} \mathrm{Li},{ }^{10} \mathrm{~B} /{ }^{9} \mathrm{Be}$, and ${ }^{7} \mathrm{Li} /{ }^{9} \mathrm{Be}$ compared with previous data and with the predictions of GALPROP. (Data from CRIS: •, Garcia-Munoz et al. 1977: O, Lukasiak et al. 1999: ム)

diffusion (Moskalenko et al., 2002), our local environment (the Local Bubble) may produce a fresh "unprocessed" nuclei component in GCR at low energy (Davis et al., 2000; Moskalenko et al., 2003b), or a more intense nucleon spectra in distant regions could yield more antiprotons and diffuse $\gamma$-rays (Strong et al., 2004). New accurate data on $\mathrm{LiBeB}$ spectra and isotopic abundances by CRIS may be used to test further the reacceleration model (Moskalenko et al., 2003a).

In our calculations we use the propagation model GALPROP as described in detail elsewhere (Strong and Moskalenko, 1998; Moskalenko et al., 2002; Strong et al., 2004). The model is designed to perform GCR propagation calculations for nuclei $(Z \leq 28)$, antiprotons, electrons and positrons, and computes $\gamma$-rays and synchrotron emission in the same framework. GALPROP solves the transport equation with a given source distribution and boundary conditions (free escape) for all GCR species. This includes a Galactic wind (convection), diffusive reacceleration, energy losses, nuclear fragmentation, radioactive decay, and production of secondary particles and isotopes.

The code includes cross-section measurements and energy dependent fitting functions (Strong and Moskalenko, 2001). The nuclear re- action network is built using the Nuclear Data Sheets. The isotopic cross section database is built using the extensive T16 Los Alamos compilation of the cross sections (Mashnik et al., 1998) and modern nuclear codes CEM2k and LAQGSM (Mashnik et al., 2004). The most important isotopic production cross sections $\left({ }^{2} \mathrm{H}\right.$, $\left.{ }^{3} \mathrm{H},{ }^{3} \mathrm{He}, \mathrm{Li}, \mathrm{Be}, \mathrm{B}, \mathrm{Al}, \mathrm{Cl}, \mathrm{Sc}, \mathrm{Ti}, \mathrm{V}, \mathrm{Mn}\right)$ are calculated using our fits to major production channels (e.g., Moskalenko et al., 2001, 2003b; Moskalenko and Mashnik, 2003). Other cross sections are calculated using phenomenological approximations by Webber et al. (1990) (code WNEWTR. FOR versions of 1993 and 2003) and/or Silberberg and Tsao (Silberberg et al., 1998) (code YIELDX_011000.FOR version of 2000) renormalized to the data where it exists. For $p A$ inelastic cross section we adapted the parametrization by Barashenkov and Polanski (code CROSEC, Barashenkov, 1993; Barashenkov and Polanski, 1994).

The propagation equation is solved numerically starting at the heaviest nucleus (i.e., ${ }^{64} \mathrm{Ni}$ ), computing all the resulting secondary source functions, and then proceeds to the nuclei with $A-1$. The procedure is repeated down to $A=1$. To account for some special $\beta^{-}$-decay cases (e.g., ${ }^{10} \mathrm{Be} \rightarrow{ }^{10} \mathrm{~B}$ ) the whole loop is repeated twice. The current version employs a full 3-dimensional spatial grid for all GCR species, but for the purposes of this study the $2 \mathrm{D}$ cylindrically symmetrical option is sufficient.

For a given size of Galactic halo, matching the propagation model predictions to the observations of GCR B/C both at low energy with CRIS data and at high energy with HEAO-3 data (Englemann et al., 1990) determines the diffusion coefficient as a function of momentum and the Alfvén speed. The halo size $4 \mathrm{kpc}$ is based on constraints set by the radioactive isotopes ${ }^{10} \mathrm{Be}$, ${ }^{26} \mathrm{Al},{ }^{36} \mathrm{Cl}$, and ${ }^{54} \mathrm{Mn}$ (Strong and Moskalenko, 2001; Moskalenko et al., 2001). Assuming a Kolmogorov spectrum of interstellar turbulence, it yields $6.5 \times 10^{28} \beta(\rho / 4 \mathrm{GV})^{\delta}\left(\mathrm{cm}^{2} \mathrm{~s}^{-1}\right)$, where $\rho$ is the rigidity, $\delta=1 / 3$, the Alfvén speed $v_{A}=35$ $\mathrm{km} \mathrm{s}^{-1}$, and the exact values of the reacceleration parameters depend mainly on the adopted crosssections. The diffusion coefficient is assumed to be independent on the spatial coordinates. While 
there is no a clear evidence that the diffusion coefficient should be distinctly different in the disk and in the halo, this minimizes the number of parameters to be determined from the data. The current state of the data on radioactive isotopes do not warrant more a complicated approach. The measured isotopes to-date $\left({ }^{10} \mathrm{Be},{ }^{26} \mathrm{Al},{ }^{36} \mathrm{Cl}\right.$, and $\left.{ }^{54} \mathrm{Mn}\right)$ all have halves lives around 1 Myrs. To test the diffusion coefficient at different distances from the Sun and in the halo, one needs accurate measurements of radioactive isotopic abundances in CRs that include isotopes with different lifetimes (e.g. $\left.{ }^{14} \mathrm{C}\right)$. Heavy elements that have large fragmentation cross sections may provide another way to test the characteristics of the local interstellar medium (Moskalenko et al., 2005).

Supernova remnants (SNR) are believed to be the primary sources of Galactic cosmic rays. Observations of X-ray (Kovama et al., 1995) and $\gamma$-ray emission (Aharonian et al., 2005, 2006) from SNR shocks reveal the presence of energetic particles thus testifying to efficient acceleration processes. The predicted spectrum of accelerated particles has power-law in rigidity with index which may vary around -2.0 (e.g., Ellison \& Cassam-Chenaï, 2005; Berezhko \& Völk, 2006). Such a hard injection spectrum poses a difficulty in reconciling the propagated spectrum with the direct CR measurements, assuming the Kolmogorov spectrum of interstellar turbulence. The latter is favored by the data (Woo \& Armstrong, 1979; Saur \& Bieber, 1999) and MHD simulations (Verma et al., 1996). In our calculations, the injection spectrum is tuned to match the spectra of primary GCR nuclei for a given propagation model. For the reacceleration model, the injection spectrum is taken as a power-law in rigidity with index 2.1 below $9 \mathrm{GV}$ and 2.42 above $9 \mathrm{GV}$, where the injection index at high energies is fixed by the rigidity dependence of the diffusion coefficient $\propto \rho^{1 / 3}$. The source abundances are tuned to match the ACE/CRIS elemental and isotopic abundances Wiedenbeck et al., 2001). Generally, the injection spectrum is model dependent: different propagation models assume a different rigidity dependence of the diffusion coefficient while the propagated spectra are tuned to the same local data. For reacceleration, this requires a change in the injection spectrum in- dex. At low energies, direct measurements of the interstellar spectrum are impossible due to the solar modulation, while indirect observations of the proton spectrum via pionic gamma rays by EGRET are not accurate enough and cannot be used to fix the spectrum below a few $\mathrm{GeV}$. The spectrum of CR nucleons below $\sim 10 \mathrm{GeV} /$ nucleon is thus tuned to the local data assuming modulation models, which are in turn, approximate and also rely on the interstellar spectrum.

With the availability of precise GCR data from CRIS, uncertainties in the fragmentation cross sections have become a significant limitation to the study of rare GCR species that are generated predominantly via spallation (Yanasak et al., 2001; Moskalenko et al., 2001). A review of current cross sections (Moskalenko and Mashnik, 2003) was undertaken for the dominant reactions involving the production of $\mathrm{LiBeB}$ as well as products decaying to LiBeB species (e.g., ${ }^{6} \mathrm{He},{ }^{10,11} \mathrm{C}$ ). For instance, $\beta^{-}$-decay of ${ }^{10} \mathrm{Be}$ contributes significantly to ${ }^{10} \mathrm{~B}$, while ${ }^{7}$ Be may produce some ${ }^{7} \mathrm{Li}$ via electron capture. Sisterson et al. (1997) have surveyed partial ${ }^{7,10}$ Be production cross sections for fragmentation of $\mathrm{O}$ on hydrogen over a range of energies $E \sim 30$ $500 \mathrm{MeV} /$ nucleon. Higher energy cross-section measurements (E $365-600 \mathrm{MeV} /$ nucleon, from Webber et al., 1990, 1998) for $\mathrm{p}+\mathrm{CNO} \rightarrow \mathrm{LiBeB}$ reactions have also been made. Michel et al. (1995) gives ${ }^{7,10} \mathrm{Be}$ production cross sections for fragmentation of $\mathrm{CNO}$ on hydrogen over a range of energies E 800-2600 MeV/nucleon. In addition, measurements for $\mathrm{He}+\mathrm{CN} \rightarrow \mathrm{BeB}$ reactions at 600 $\mathrm{MeV} /$ nucleon (Webber et al., 1990) and $\alpha+\alpha \rightarrow$ $\mathrm{Li},{ }^{7} \mathrm{Be}$ at $60-160 \mathrm{MeV}$ (Mercer et al., 1997) are available.

$\mathrm{Li}, \mathrm{Be}$, and B in cosmic rays are mainly produced from the interaction of CNO nuclei with interstellar hydrogen. Some of the dominant reactions, such as ${ }^{12} \mathrm{C} \rightarrow{ }^{6} \mathrm{Li},{ }^{7,9,10} \mathrm{Be},{ }^{11} \mathrm{~B},{ }^{14} \mathrm{~N} \rightarrow{ }^{7} \mathrm{Be},{ }^{16} \mathrm{O} \rightarrow$ ${ }^{6} \mathrm{Li},{ }^{7,10} \mathrm{Be},{ }^{9,11} \mathrm{~B}$, are constrained by the data. The cross sections for the production of boron are better known than for the lighter isotopes of beryllium and lithium, since the main contribution to boron production is from $\mathrm{CNO}$ and ${ }^{11} \mathrm{~B} \rightarrow \mathrm{B}$. Thus the model parameters are rather tightly constrained by the measured $\mathrm{B} / \mathrm{C}$ ratio at low and high energies. However, some channels of Be and Li show a 
spread in the data between $10-20 \%$ or greater. For example, ${ }^{7} \mathrm{Be}$ production by $\mathrm{CNO}$ nuclei shows a spread by a factor of $\sim 2$ at energies below a few $100 \mathrm{MeV} /$ nucleon. Some important channels are represented by one or few data points in a narrow energy range (e.g., ${ }^{11} \mathrm{~B}, \mathrm{C}, \mathrm{O} \rightarrow{ }^{10} \mathrm{~B},{ }^{11} \mathrm{~B} \rightarrow{ }^{9} \mathrm{Be}$ ), while some are not measured at all (e.g., ${ }^{10} \mathrm{~B}, \mathrm{~N}$ $\left.\rightarrow{ }^{9} \mathrm{Be}, \mathrm{B}, \mathrm{Be} \rightarrow{ }^{7} \mathrm{Be}\right)$. The production of $\mathrm{Li}$ isotopes is particularly poorly measured. There are only a few data points for Li production by $\mathrm{CNO}$ nuclei. The data on $\mathrm{Li}$ isotopic production by ${ }^{7} \mathrm{Li}$, Be, B nuclei are absent, while a contribution from spallation of these isotopes may exceed $\sim 35 \%$. See Moskalenko and Mashnik (2003) for more details.

When there is no data, the production cross sections are calculated using the semi-empirical parametrizations. This translates to about a $15 \%$ overall uncertainty in the isotopic production ( $\sim 20 \%$ for $\mathrm{Li}$ ), given that typical cross section uncertainties of semi-empirical parametrizations are of the order of $\sim 50 \%$.

The solid curves in Figs. 1 and 2 correspond to GALPROP predictions modulated assuming a modulation parameter of $550 \mathrm{MV}$, corresponding to the time period covered in this study (Jan. 1, 1998 to Jan. 23, 1999). The dashed curves in Fig. 1a refer to modulated GALPROP predictions with $\phi=800 \mathrm{MV}$, appropriate for the modulation level during HEAO-3 observations. The model is tuned to match the observations of $\mathrm{C}$ and $\mathrm{B}$ intensities and the $\mathrm{B} / \mathrm{C}$ ratio from CRIS (Fig. 1a/b). However, Be and $\mathrm{Li}$ intensities and the ratios $\mathrm{Li} / \mathrm{C}$ and $\mathrm{Be} / \mathrm{C}$ are $\sim 10-20 \%$ lower than predicted by the model, although still consistent within the uncertainty determined for the propagation calculation (shown as the hatched region). The isotopic ratios shown in Fig. 2 are also in good agreement with the model predictions. The model uncertainties are mostly due to uncertainties in the cross sections of tertiary interactions such as the production of Be and $\mathrm{Li}$ from isotopes of $\mathrm{Li}, \mathrm{Be}$, and $\mathrm{B}$ as discussed above. Further work is required to pin down these uncertainties.

\section{Conclusion}

We have made GCR LiBeB abundance measurements using the CRIS instrument during near solar minimum conditions in 1998-1999. The isotopic ratios of $\mathrm{LiBeB}$ are in agreement with previous measurements of GCR light isotopes, particularly for those experiments acquiring data during periods of solar modulation levels similar to the modulation levels in 1998-1999 covered by CRIS. The reacceleration model gives a satisfactory prediction for GCR primary and secondary species with $Z \geq 3$, and shows good agreement with relative isotopic abundances (Jones et al., 2001b; Moskalenko et al., 2002). In some cases, notably $(p, \alpha)+(\mathrm{Li}, \mathrm{Be}, \mathrm{B}) \rightarrow \mathrm{Be}, \mathrm{Li}$, a lack of cross-section measurements limits the reliability of model predictions. Understanding the subtle differences between the model predictions and experimental data now hinges on more precise cross section measurements, especially for reactions involving the production of the light isotopes of LiBeB.

This research was supported by NASA at the NASA/Goddard Space Flight Center, the California Institute of Technology, (grant NAG5-6912), the Jet Propulsion Laboratory, and Washington University. I.V.M. acknowledges partial support from NASA Astrophysics Theory Program (ATP) grant and NASA Astronomy and Physics Research and Analysis Program (APRA) grant.

References

Aharonian, F., Akhperjanian, A.G., Bazer-Bachi, A.R., et al., Detection of TeV gamma-ray emission from the shell-type supernova remnant RX J0852.0-4622 with HESS., Astron. \& Astrophys. 437, L7-L10, 2005

Aharonian, F., Akhperjanian, A.G., Bazer-Bachi, A.R., et al., A detailed spectral and morphological study of the gamma-ray supernova remnant RX J1713.7-3949 with HESS., Astron. \& Astrophys. 449, 223-242, 2006

Barashenkov, V. S., Cross-Sections of Interactions of Particles and Nuclei with Nuclei, (Dubna: 
Joint Institute for Nuclear Research) , 1993

Barashenkov V. S., \& Polanski A., Electronic Guide for Nuclear Cross-Sections, Comm. JINR E2-94-417 (Dubna: Joint Institute for Nuclear Research), 1994

Berezhko, E. G. \& Völk, H. J., Theory of cosmic ray production in the supernova remnant $\mathrm{RX}$ J1713.7-3946., Astron. \& Astrophys. 451, 981990,2006

Buckley, J., Dwyer, J., Muller, D., et al., A new measurement of the flux of the light cosmic-ray nuclei at high energies., Astrophys. J. 429, 736747, 1994

Buffington, A., Orth, C.D., Mast, T.S., A measurement of the cosmic-ray beryllium isotopes from 200 to $1500 \mathrm{MeV}$ per nucleon., Astrophys. J. 226, 355-371, 1978

Chapell, J.H., Webber, W.R., High energy cosmic ray charge and energy spectra measurements., Proc. 17th ICRC (Paris) 2, 59-63, 1981

Connell, J.J., Cosmic-ray composition as observed by Ulysses., Space Sci. Rev. 99, 41-50, 2001

Connell, J.J., Galactic Cosmic-Ray Confinement Time: Ulysses High Energy Telescope Measurements of the Secondary Radionuclide 10Be., Astrophys. J. 501, L59-L62, 1998

Davis, A.J., Mewaldt, R.A., Binns, W.R., et al., On the low energy decrease in galactic cosmic ray secondary/primary ratios., AIP Conf. Proc. (Indian Wells, California) 528, 421-424, 2000

Davis, A.J., Mewaldt, R.A., Binns, W.R., et al., The evolution of galactic cosmic ray element spectra from solar minimum to solar maximum: ACE measurements., Proc. 27th ICRC (Hamburg) 10, 3971-3974, 2001

de Nolfo, G.A., Yanasak, N.E., Binns, W.R., et al., Cosmic-ray Lithium, Beryllium, and Boron Isotopes from ACE/CRIS., Proc. 27th ICRC (Hamburg), 2, 1667-1670, 2001

de Nolfo, G.A., Yanasak, N.E., Binns, W.R., et al., New measurements of $\mathrm{Li}, \mathrm{Be}$, and B isotopes as a test of cosmic-ray transport models., Proc. 28th ICRC (Tsukuba), 2, 1777-1780, 2003

Dwyer, R., The mean mass of the abundant cosmicray nuclei from boron to silicon at $1.2 \mathrm{GeV}$ per atomic mass unit., Astrophys. J. 224, 691-707, 1978

Duvernois, M.A., Thayer, M.R., The elemental composition of the galactic cosmic-ray source: Ulysses High Energy Telescope Results., Astrophys. J. 465, 982-984, 1996

Ellison, D. C. \& Cassam-Chenaï, G., Radio and Xray profiles in supernova remnants undergoing efficient cosmic ray production., Astrophys. J. 632, 920-931, 2005

Englemann, J.J., Ferrando, P., Soutoul, A., et al., Charge composition and energy spectra of cosmic-ray nuclei for elements from $\mathrm{Be}$ to $\mathrm{Ni}$ results from HEAO-3-C2., Astron. \& Astrophys. 233, 96-111, 1990

Fisher, A.J., Hagen, F.A., Maehl, R.C., et al., The isotopic composition of cosmic rays with $\mathrm{Z}$ between 5 and 26., Astrophys. J. 205, 938-946, 1976

Fisk, L.A., Solar modulation of galactic cosmic rays: 2., J. Geophys. Res. 76, 221-226, 1971

Freier, P.S., Ney, E.P., Waddington, C.J., Lithium, beryllium, and boron in the primary cosmic radiation., Phys. Rev. Lett. 113, 921-927, 1959

Garcia-Munoz, M., Mason, G.M., Simpson, J.A., The age of the galactic cosmic rays derived from the abundance of Be-10., Astrophys. J. 217, 859877, 1977

Garcia-Munoz, M., Simpson, J.A., Wefel, J.P., The propagation lifetime of galactic cosmic rays determined from the measurement of the beryllium isotopes., Proc. 17th ICRC (Paris) 2, 72-75, 1981

Garcia-Munoz, M., Simpson, J.A., Guzik, T.G., et al., Cosmic-ray propagation in the galaxy and in the heliosphere-the pathlength distribution at low energies., Astrophys. J. Suppl. 64, 269-304, 1987

Gleeson, L.J., Axford, W.I.,, Solar modulation of galactic cosmic rays., Astrophys. J. 154, 10111026, 1968

Hagen, F.A., Fisher, A.J., Ormes, J.F., Be-10 abundance and the age of cosmic rays- $\mathrm{A}$ balloon experiment., Astrophys. J. 212, 262-277, 1977

Jones, F.C., Lukasiak, A., Ptuskin, V., Webber, W., K-capture cosmic ray secondaries and reacceleration., Adv. Space Res. 27, 737-741, 2001a

Jones, F.C., Lukasiak, A., Ptuskin, V., Webber, W., The Modified Weighted Slab Technique: Models and Results., Astrophys. J. 547, 264-271, $2001 b$

Julliusson, E., Charge composition and energy spectra of cosmic-ray nuclei at energies above 20 
GeV per nucleon., Astrophys. J. 191, 331-348, 1974

Koyama, K., Petre, R., Gotthelf, E.V., et al., Evidence for shock accleration of high energy electrons in the supernova remnant SN:1006., Nature 378, 255-258, 1995

Krombel, K. E., Wiedenbeck, M. E., Isotopic composition of cosmic-ray boron and nitrogen., Astrophys. J. 328, 940-953, 1988

Lezniak, J.A., Webber, W.R., The charge composition and energy spectra of cosmic-ray nuclei from $3000 \mathrm{MeV}$ per nucleon to $50 \mathrm{GeV}$ per nucleon., Astrophys. J. 223, 676-696, 1978

Lukasiak, A., McDonald, F.B., Webber, W.R., Voyager measurements of the isotopic composition of Li, Be, and B nuclei., Proc. 26th ICRC (Salt Lake City) 3, 389-391, 1999

Maehl, R.C., Ormes, J.F., Fisher, A.J., Hagen, F.A., Energy spectra of cosmic-ray nuclei- Z of 4to 26 and $\mathrm{E}$ of .3 to $2 \mathrm{GeV} / \mathrm{amu}$., Astrophys. Space Sci. 47, 163-184, 1977

Mashnik, S. G., Sierk, A. J., Van Riper, K. A., \& Wilson, W. B., Production and Validation of Isotope Production Cross Section Libraries for Neutrons and Protons to $1.7 \mathrm{GeV}$., Proc. 4th Workshop on Simulating Accelerator Radiation Environments, ed. Gabriel, T. A. (Oak Ridge: ORNL), p.151, 1998 (nucl-th/9812071)

Mashnik, S.G., Gudima, K.K., Moskalenko, I.V., et al., CEM2K and LAQGSM codes as event generators for space radiation shielding and cosmicray propagation applications., Adv. Space Res. 34, 1288-1296, 2004

Mercer, D.J., Austin, S.M., Glagola, B.G., suggested correction to ${ }^{6} \mathrm{He},{ }^{6,7} \mathrm{Li}$, and ${ }^{7} \mathrm{Be}$ production cross sections in $\alpha+\alpha$ reactions between 60 adn 160 MeV., Phys. Rev. C 55, 946-949, 1997

Mewaldt, R.A., Spalding, J.D., Stone, E.C., Vogt, R.E., The isotopic composition of cosmic ray $B$, C, N, and O nuclei., Astrophys. J. 251, L27-L31, 1981

Michel, R., Gloris, M., Lange, H.J., et al., Nuclide production by proton-induced reactions on elements $\left(6 j Z_{i} 29\right)$ in the energy range from 800 to 2600 MeV., Nucl. Instr. Meth. B 103, 183-222, 1995

Moskalenko, I.V., Mashnik, S.G., Strong, A.W., New calculation of radioactive secondaries in cosmic rays., Proc. 27th ICRC (Hamburg), 1836-1839, 2001

Moskalenko, I.V., Strong, A.W., Ormes, J.F., Potgieter, M.S., Secondary antiprotons and propagation of cosmic rays in the Galaxy and heliosphere., Astrophys. J. 565, 280-296, 2002

Moskalenko, I.V., Mashnik, S.G., Evaluation of Production Cross Sections of Li, Be, B in CR., Proc. 28th ICRC (Tsukuba) 2, 1969-1972, 2003

Moskalenko, I.V., Strong, A.W., Mashnik, S.G., Jones, F.C., Propagation of Light Elements in the Galaxy., Proc. 28th ICRC (Tsukuba) , 19171920, 2003a

Moskalenko, I.V., Strong, A.W., Mashnik, S.G., Ormes, J.F., Challenging cosmic ray propagation with antiprotons. Evidence for a 'fresh' nuclei component?, Astrophys. J. 586, 1050-1066, $2003 \mathrm{~b}$

Moskalenko, I.V., Strong, A.W., Mashnik, S.G., Propagation of cosmic rays: nuclear physics in cosmic-ray studies., AIP Conf. Proc. (Sante Fe) 769, 1612-1617, 2005

Muller, D., Swordy, S.P., L'Heureux, J., et al., Energy spectra and composition of primary cosmic rays., Astrophys. J. 374, 356-365, 1991

Niebur, S.M., Scott, L.M., Wiedenbeck, M.E., et al., Cosmic ray energy loss in the heliosphere: direct evidence from electron-capture-decay secondary isotopes., J. Geophys. Res. 108, 80338042, 2003

Orth, C.D., Buffington, A., Smoot, G., Mast, T.S., Abundances and spectra for cosmic-ray nuclei from $\mathrm{Li}$ to $\mathrm{Fe}$ for 2 to $150 \mathrm{GeV}$ per nucleon., Astrophys. J. 226, 1147-1161, 1978

Saur, J., \& Bieber, J. W., Geometry of low frequency solar wind magnetic turbulence: evidence for radially aligned Alfvenic fluctuations., J. Geophys. Res. 104, 9975-9988, 1999

Seo, E.S., Ptuskin, V.S., Stochastic reacceleration of cosmic rays in the interstellar medium., Astrophys. J. 431, 705-714, 1994

Silberberg, R., Tsao, C. H., \& Barghouty, A. F., Updated Partial Cross Sections of ProtonNucleus Reactions, Astrophys. J. 501, 911-919, 1998

Simon, M., Speigelhauer, H., Schmidt, W.K.H., et al., Energy spectra of cosmic-ray nuclei to above $100 \mathrm{GeV}$ per nucleon., Astrophys. J. 239, 712- 
724,1980

Sisterson, J. M., Kim, K., Beverding, A., et al., Measurement of proton production cross sections of $10 \mathrm{Be}$ and $26 \mathrm{Al}$ from elements found in lunar rocks., Nucl. Instr. Meth. B 123, 324-329, 1997

Stone, E.C., Cohen, C.M.S., Cook, W.R., et al., The Cosmic Ray Isotope Spectrometer for The Advanced Composition Explorer., Space Sci. Rev. 86, 285-356, 1998

Strong, A.W., Moskalenko, I.V., Propagation of cosmic-ray nucleons in the Galaxy, Astrophys. J. 509, 212-228, 1998

Strong, A.W., Moskalenko, I.V., Models for Galactic cosmic-ray propagation, Adv. Space Res. 27, 717-726, 2001

Strong, A.W., Moskalenko, I.V., Reimer, O., Diffuse Galactic continuum gamma rays. A model compatible with EGRET data and cosmic-ray measurements, Astrophys. J. 613, 962-976, 2004

Verma, M. K., Roberts, D. A., Goldstein, M. L., Ghosh, S., \& Stribling, W. T., A numerical study of nonlinear cascade of energy in magnetohydrodynamic turbulence., J. Geophys. Res. 101, 21619-21625, 1996

Webber, W.R., Lukasiak, A., McDonald, F.B., Voyager Measurements of the charge and composition of cosmic-ray $\mathrm{Li}, \mathrm{Be}$, and $\mathrm{B}$ nuclei and implications for the production in their galaxy., Astrophys. J. 568, 210-215, 2002

Webber, W.R., Kish, J.C., Rockstroh, J.M., et al., Production cross sections of fragmentations from beams of 400-650 MeV per nucleon 9Be, $11 \mathrm{~B}, 12 \mathrm{C}, 14 \mathrm{~N}, 16 \mathrm{O}, 20 \mathrm{Ne}, 56 \mathrm{Fe}$, and $58 \mathrm{Ni}$ nulcei interacting in a liquid hydrogen target. II. Isotopic cross sections of fragments., Astrophys. J. 508, 949-958, 1998

Webber, W.R., Kish, J.C., Schrier, D.A., Formula for calculating partial cross sections for nuclear reactions of nuclei with $E \geq 200 \mathrm{MeV} /$ nucleon in hydrogen targets., Phys. Rev. C 41, 566-571, 1990

Webber, W.R., Lezniak, J.A., Kish, J.C., Simpson, G.A., A measurement of the abundance of cosmic-ray Be-10 and its implication for the cosmic ray age., Astrophys. Lett. 18, 125-132, 1977

Webber, W.R., Damle, S.V., Kish, J.C., Studies of the chemical composition of cosmic rays with
$\mathrm{Z}=3-30$ at high and low energies., Astrophys. Space Sci. 15, 245-271, 1972

Wiedenbeck, M.E., Greiner, D.E., A cosmic ray age based on the abundance of Be-10., Astrophys. J. 239, L139-L142, 1980

Wiedenbeck, M.E., Yanasak, N.E., Cummings, A.C., et al., The origin of primary cosmic rays: constraints from ACE elemental and isotopic composition observations., Space Sci. Rev. 99, 15-26, 2001

Woo, R., \& Armstrong, J.W., Spacecraft radio scattering observations of the power spectrum of electron-density fluctuations in the solar-wind., J. Geophys. Res. 84, 7288-7296, 1979

Yanasak, N. E., Wiedenbeck, M.E., Mewaldt, R.A., et al., Measurement of the secondary radionuclides ${ }^{10} \mathrm{Be},{ }^{26} \mathrm{Al},{ }^{36} \mathrm{Cl},{ }^{54} \mathrm{Mn}$, and ${ }^{14} \mathrm{C}$ and implications for the galactic cosmic-ray age., Astrophys. J. 563-792, 768, 2001 